\title{
Blow Up and Global Existence for a Nonlinear Viscoelastic Wave Equation with Strong Damping and Nonlinear Damping and Source terms
}

\author{
Liang Guo, Zhaoqin Yuan, Guoguang Lin \\ Department of Mathematics, Yunnan University, Kunming, China \\ Email: guoliang142857@163.com, yuanzq091@163.com, gglin@ynu.edu.cn
}

Received 30 March 2015; accepted 12 May 2015; published 14 May 2015

Copyright (C) 2015 by authors and Scientific Research Publishing Inc.

This work is licensed under the Creative Commons Attribution International License (CC BY).

http://creativecommons.org/licenses/by/4.0/

c) (i) Open Access

\begin{abstract}
In this paper, we consider an initial-boundary value problem for a nonlinear viscoelastic wave equation with strong damping, nonlinear damping and source terms. We proved a blow up result for the solution with negative initial energy if $p>m$, and a global result for $p \leq m$.
\end{abstract}

\section{Keywords}

\section{Viscoelastic Equation, Blow Up, Global Existence}

\section{Introduction}

A purely elastic material has a capacity to store mechanical energy with no dissipation (of the energy). A complete opposite to an elastic material is a purely viscous material. The important thing about viscous materials is that when the force is removed it does not return to its original shape. Materials which are outside the scope of these two theories will be those for which some, but not all, of the work done to deform them can be recovered. Such materials possess a capacity of storage and dissipation of mechanical energy. This is the case for viscoelastic material. The dynamic properties of viscoelastic materials are of great importance and interest as they appear in many applications to natural sciences. Many authors have given attention to this problem for quite a long time, especially in the last two decades, and have made a lot of progress.

In [1], Messaoudi considered the following initial-boundary value problem:

$$
\left\{\begin{array}{l}
u_{t t}-\Delta u+\int_{0}^{t} g(t-s) \Delta u(s) \mathrm{d} s+\left|u_{t}\right|^{m-2} u_{t}=|u|^{p-2} u, \quad \text { in } \Omega \times(0, \infty) \\
u(x, t)=0, \quad x \in \partial \Omega, t \geq 0 ; \\
u(x, 0)=u_{0}(x), \quad u_{t}(x, 0)=u_{1}(x), \quad x \in \Omega .
\end{array}\right.
$$

How to cite this paper: Guo, L., Yuan, Z.Q. and Lin, G.G. (2015) Blow Up and Global Existence for a Nonlinear Viscoelastic Wave Equation with Strong Damping and Nonlinear Damping and Source terms. Applied Mathematics, 6, 806-816. 
where $\Omega$ was a bounded domain of $R^{n}(n \geq 1)$ with a smooth boundary $\partial \Omega, m \geq 2, p>2$, and $g: R^{+} \rightarrow R^{+}$ was a positive nonincreasing function. He proved a blow up result for the solution with negative initial energy if $p>m$, and a global result for $p \leq m$. This result was later improved by Messaoudi [2], to certain solutions with positive initial energy. A similar result was also obtained by $\mathrm{Wu}$ [3] using a different method.

For the problem (1.1) in $R^{n}$ and with $m=2$, concerning Cauchy problems, Kafini and Messaoudi [4] established a blow up result for the problem

$$
\begin{cases}u_{t t}-\Delta u+\int_{0}^{t} g(t-s) \Delta u(s) \mathrm{d} s+u_{t}=|u|^{p-2} u, & x \in R^{n}, t>0 \\ u(x, 0)=u_{0}(x), \quad u_{t}(x, 0)=u_{1}(x), & x \in R^{n} .\end{cases}
$$

where $g$ satisfied $\int_{0}^{+\infty} g(s) \mathrm{d} s<(2 p-4) /(2 p-3)$ and the initial data were compactly supported with negative energy such that $\int u_{0} u_{1} \mathrm{~d} x \geq 0$.

In the absence of the viscoelastic term $(g=0)$, the problem has been extensively studied and results concerning existence and nonexistence have been established. In bounded domains, for the equation

$$
u_{t t}-\Delta u+a\left|u_{t}\right|^{m-2} u_{t}=b|u|^{p-2} u, \quad \text { in } \Omega \times(0, \infty)
$$

$m \geq 2, \quad p>2, a, b \geq 0$, it is well known that, for $a=0$, the source term $b|u|^{p-2} u$ causes finite time blow up of solutions with negative initial energy (see [5]). In contrast, for $b=0$, the damping term $a\left|u_{t}\right|^{m-2} u_{t}$ assures global existence for arbitrary initial data (see [6]). The case of linear damping $(m=2)$ and nonlinear source has been first considered by Levine [7] [8]. He showed that solutions with negative initial energy blew up in finite time. Furthermore, the interaction between the nonlinear damping $(m>2)$ and the source terms was studied by Georgiev and Todorova [9], for a bounded domain with Dirichlet boundary conditions. For the same problem, Messaoudi [10] extended the blow up result to solutions with negative initial energy.

In [11], Berrimi and Messaoudi considered

$$
\begin{cases}u_{t t}-\Delta u+\int_{0}^{t} g(t-s) \Delta u(s) \mathrm{d} s=|u|^{p-2} u, & x \in \Omega, t>0 \\ u(x, t)=0, & x \in \partial \Omega, t \geq 0 \\ u(x, 0)=u_{0}(x), \quad u_{t}(x, 0)=u_{1}(x), & x \in \Omega\end{cases}
$$

in a bounded domain and $p>2$. They established a local existence result and showed that the local solution was global and decays uniformly if the initial data were small enough.

In [12], Song and Xue considered with the following viscoelastic equation with strong damping:

$$
\begin{cases}u_{t t}-\Delta u+\int_{0}^{t} g(t-s) \Delta u(s) \mathrm{d} s-\Delta u_{t}=|u|^{p-2} u, & x \in \Omega, t \in[0, T] \\ u(x, t)=0, & x \in \partial \Omega, t \in[0, T] \\ u(x, 0)=u_{0}(x), \quad u_{t}(x, 0)=u_{1}(x), & x \in \Omega .\end{cases}
$$

where $\Omega$ was a bounded domain of $R^{n}(n \geq 1)$ with a smooth boundary $\partial \Omega, m \geq 2, p>2$, and $g: R^{+} \rightarrow R^{+}$was a positive nonincreasing function. They showed, under suitable conditions on $g$, that there were solutions of (1.5) with arbitrarily high initial energy that blow up in a finite time. For the same problem (1.5), in [13], Song and Zhong showed that there were solutions of (1.5) with positive initial energy that blew up in finite time. For more related works, we refer the reader to [14]-[18].

In this work, we intend to study the following initial-boundary value problem:

$$
\begin{cases}u_{t t}-\Delta u+\int_{0}^{+\infty} g(s) \Delta u(t-s) \mathrm{d} s-\varepsilon_{1} \Delta u_{t}+\varepsilon_{2}\left|u_{t}\right|^{m-2} u_{t}=\varepsilon_{3}|u|^{p-2} u, & x \in \Omega, \quad t>0 ; \\ u(x, t)=0, & x \in \Omega, \quad t>0 ; \\ u(x, 0)=u_{0}(x), \quad u_{t}(x, 0)=u_{1}(x), & x \in \Omega .\end{cases}
$$


where $\Omega \subset R^{n}(n \geq 1)$ is a bounded domain with a smooth boundary $\partial \Omega, m \geq 2, p>2, \varepsilon_{1}, \varepsilon_{2} \geq 0, \varepsilon_{3}>0$, for the problem (1.6), the memory term $\int_{0}^{+\infty} g(s) \Delta u(t-s) \mathrm{d} s$ (see [19] [20]) replaces $\int_{0}^{t} g(t-s) \Delta u(s) \mathrm{d} s$, and we consider the strong damping term $-\varepsilon_{1} \Delta u_{t}$ and the nonlinear damping term $\varepsilon_{2}\left|u_{t}\right|^{m-2} u_{t}$.

Now, we shall add a new variable $\eta=\eta(s)=\eta^{t}(x, s)$ to the system which corresponds to the relative displacement history. Let us define

$$
\eta=\eta(s)=\eta^{t}(x, s)=u(x, t)-u(x, t-s)
$$

A direct computation yields

$$
\eta_{t}(s)=-\eta_{s}(s)+u_{t}(t)
$$

Thus, the original memory term can be written as

$$
\int_{0}^{+\infty} g(s) \Delta u(t-s) \mathrm{d} s=\int_{0}^{+\infty} g(s) \mathrm{d} s \cdot \Delta u-\int_{0}^{+\infty} g(s) \Delta \eta(s) \mathrm{d} s
$$

and we get a new system

$$
\begin{gathered}
u_{t t}-\left(1-\int_{0}^{+\infty} g(s) \mathrm{d} s\right) \Delta u-\varepsilon_{1} \Delta u_{t}-\int_{0}^{+\infty} g(s) \Delta \eta(s) \mathrm{d} s+\varepsilon_{2}\left|u_{t}\right|^{m-2} u_{t}=\varepsilon_{3}|u|^{p-2} u \\
\eta_{t}=-\eta_{s}+u_{t}
\end{gathered}
$$

with the initial conditions

$$
u(x, 0)=u_{0}(x), u_{t}(x, 0)=u_{1}(x), \eta(0)=\eta^{t}(x, 0)=0, \quad x \in \Omega
$$

and boundary conditions

$$
u(x, t)=0, \quad x \in \partial \Omega, t \geq 0
$$

The paper is organized as follows. In Section 2, we first prove the blow up result, and then in Section 3, we prove the global existence result.

For convenience, we denote the norm and scalar product in $L^{2}(\Omega)$ by $\|\cdot\|$ and $(\cdot, \cdot)$, and let $V=H^{1}(\Omega)$. $C$ denotes a general positive constant, which may be different in different estimates.

\section{Blow Up}

In this section, we present some materials needed in the proof of our results, state a local existence result, which can be established, combining the argument of [21], and prove our main result. For this reason, we assume that

(G1) $g: R^{+} \rightarrow R^{+}$is a differentiable function satisfying $1-\int_{0}^{+\infty} g(s) \mathrm{d} s=l>0$;

(G2) $g(s) \geq 0, g^{\prime}(s) \leq 0, \quad \forall s \in R^{+}$;

(G3) There exists a constant $\xi>0$ such that $g^{\prime}(s)+\xi g(s) \leq 0, \forall s \in R^{+}$;

We start with a local existence theorem which can be established by the Faedo-Galerkin methods. The interested readers are referred to Cavalcanti, Domingos Cavalcanti and Soriano [22] for details:

Theorem 2.1. Assume (G1) holds. Let $m \geq 2$ and

$$
\begin{cases}2<p \leq \frac{2 n-2}{n-2}, & n \geq 3 \\ p \geq 2, & n=1,2 .\end{cases}
$$

Then for any initial data

$$
u_{0} \in H_{0}^{1}(\Omega), \quad u_{1} \in L^{2}(\Omega),
$$

with compact support, problem (1.10) has a unique solution

$$
u \in C\left([0, T] ; H_{0}^{1}(\Omega)\right) \cap C^{1}\left([0, T] ; L^{2}(\Omega)\right)
$$

for some $T>0$. 
Lemma 2.2. Assume (G1), (G2), (G3) and (2.1) hold. Let $u(t)$ be a solution of (1.10), then $E(t)$ is nonincreasing, that is

$$
E^{\prime}(t) \leq-\varepsilon_{1}\left\|\nabla u_{t}\right\|_{2}^{2}-\frac{\xi}{2}\|\eta\|_{g, V}^{2}-\varepsilon_{2}\left\|u_{t}\right\|_{m}^{m} \leq 0
$$

where

$$
E(t)=\frac{1}{2}\left\|u_{t}\right\|_{2}^{2}+\frac{1}{2}\left(1-\int_{0}^{+\infty} g(s) \mathrm{d} s\right)\|\nabla u\|_{2}^{2}+\frac{1}{2}\|\eta\|_{g, V}^{2}-\frac{\varepsilon_{3}}{p}\|u\|_{p}^{p} .
$$

Proof. By multiplying the Equation in (1.10) by $u_{t}$ and intergrating over $\Omega$, we get

$$
\begin{aligned}
& \frac{1}{2} \frac{\mathrm{d}}{\mathrm{d} t}\left\|u_{t}\right\|_{2}^{2}+\frac{1}{2}\left(1-\int_{0}^{+\infty} g(s) \mathrm{d} s\right) \frac{\mathrm{d}}{\mathrm{d} t}\|\nabla u\|_{2}^{2}+\varepsilon_{1}\left\|\nabla u_{t}\right\|_{2}^{2} \\
& +\int_{0}^{+\infty} g(s) \int_{\Omega} \nabla \eta(s) \cdot \nabla u_{t} \mathrm{~d} x \mathrm{~d} s+\varepsilon_{2}\left\|u_{t}\right\|_{m}^{m}-\frac{\varepsilon_{3}}{p} \frac{\mathrm{d}}{\mathrm{d} t}\|u\|_{p}^{p}=0
\end{aligned}
$$

For the fourth term on the left side (2.4), by using (1.11), (G2) and (G3), we have

$$
\begin{aligned}
\int_{0}^{+\infty} g(s) \int_{\Omega} \nabla \eta(s) \cdot \nabla u_{t} \mathrm{~d} x \mathrm{~d} s & =\int_{0}^{+\infty} g(s) \int_{\Omega} \nabla \eta(s) \cdot\left(\nabla \eta_{t}+\nabla \eta_{s}\right) \mathrm{d} x \mathrm{~d} s \\
& =\int_{0}^{+\infty} g(s) \frac{1}{2} \frac{\mathrm{d}}{\mathrm{d} t}\|\nabla \eta\|_{2}^{2} \mathrm{~d} s+\int_{0}^{+\infty} g(s) \mathrm{d} \frac{1}{2}\|\nabla \eta\|_{2}^{2} \\
& \geq \frac{1}{2} \frac{\mathrm{d}}{\mathrm{d} t}\|\eta\|_{g, V}^{2}+\frac{\xi}{2}\|\eta\|_{g, V}^{2},
\end{aligned}
$$

where

$$
\|\eta\|_{g, V}^{2}=\int_{0}^{+\infty} g(s)\|\nabla \eta(s)\|_{2}^{2} \mathrm{~d} s
$$

Then, we obtain

$$
\begin{aligned}
& \frac{\mathrm{d}}{\mathrm{d} t}\left[\frac{1}{2}\left\|u_{t}\right\|_{2}^{2}+\frac{1}{2}\left(1-\int_{0}^{+\infty} g(s) \mathrm{d} s\right)\|\nabla u\|_{2}^{2}+\frac{1}{2}\|\eta\|_{g, V}^{2}-\frac{\varepsilon_{3}}{p} \frac{\mathrm{d}}{\mathrm{d} t}\|u\|_{p}^{p}\right] \\
& +\varepsilon_{1}\left\|\nabla u_{t}\right\|_{2}^{2}+\frac{\xi}{2}\|\eta\|_{g, V}^{2}+\varepsilon_{2}\left\|u_{t}\right\|_{m}^{m} \leq 0
\end{aligned}
$$

So, we have

$$
E^{\prime}(t) \leq-\varepsilon_{1}\left\|\nabla u_{t}\right\|_{2}^{2}-\frac{\xi}{2}\|\eta\|_{g, V}^{2}-\varepsilon_{2}\left\|u_{t}\right\|_{m}^{m} \leq 0
$$

where

$$
E(t)=\frac{1}{2}\left\|u_{t}\right\|_{2}^{2}+\frac{1}{2}\left(1-\int_{0}^{+\infty} g(s) \mathrm{d} s\right)\|\nabla u\|_{2}^{2}+\frac{1}{2}\|\eta\|_{g, V}^{2}-\frac{\varepsilon_{3}}{p}\|u\|_{p}^{p}
$$

Our main result reads as follows.

Lemma 2.3. Suppose that (2.1) holds. Then there exists a positive constant $C>1$ such that

$$
\|u\|_{p}^{s} \leq C\left(\|\nabla u\|_{2}^{2}+\|u\|_{2}^{2}+\|u\|_{p}^{p}\right)
$$

for any $u \in H_{0}^{1}(\Omega)$ and $2 \leq s \leq p$.

Proof. If $\|u\|_{p} \leq 1$, by Sobolev embedding theorem Young's inequality, then we have

$$
\|u\|_{p}^{s} \leq\|u\|_{p}^{2} \leq C\left(\|\nabla u\|_{2}^{\alpha}\|u\|_{2}^{1-\alpha}\right)^{2} \leq C\left(\|\nabla u\|_{2}^{2}+\|u\|_{2}^{2}\right)
$$

So, we obtain

$$
\|u\|_{p}^{s} \leq C\left(\|\nabla u\|_{2}^{2}+\|u\|_{2}^{2}+\|u\|_{p}^{p}\right)
$$


If $\|u\|_{p}>1$, then

$$
\|u\|_{p}^{s} \leq\|u\|_{p}^{p} \leq C\left(\|\nabla u\|_{2}^{2}+\|u\|_{2}^{2}+\|u\|_{p}^{p}\right)
$$

Therefore (2.9) follows.

We get

$$
H(t)=-E(t)
$$

and use, throughout this paper, $C$ to denote a generic positive constant.

As a result of (2.3) and (2.5), we have

Corollary 2.4. Suppose that (2.1) holds. Then, we have

$$
\|u\|_{p}^{s} \leq C\left(-H(t)-\left\|u_{t}\right\|_{2}^{2}-\|\eta\|_{g, V}^{2}+\|u\|_{p}^{p}\right), \quad t \in[0, T)
$$

for any $u \in H_{0}^{1}(\Omega)$ and $2 \leq s \leq p$.

Lemma 2.5. ( $C_{p}$ inequality) Let $a, b$ is arbitrary real, then we have

$$
(|a|+|b|)^{p} \leq C_{p}\left(|a|^{p}+|b|^{p}\right)
$$

where

$$
C_{p}= \begin{cases}1, & 0<p \leq 1 \\ 2^{p-1}, & p>1 .\end{cases}
$$

Proof. We set $x=\frac{|a|}{|b|}$, that is to proof

$$
f(x)=\frac{(1+x)^{p}}{\left(1+x^{p}\right)} \leq C_{p}
$$

By taking a derivative of $f(x)$, we obtain

$$
f^{\prime}(x)=\frac{p(1+x)^{p-1}\left(1-x^{p-1}\right)}{\left(1+x^{p}\right)^{2}}
$$

If $0<p \leq 1$, then we know $f(x)$ is monotone decreasing on $(0,1]$ and monotone increasing on $[1,+\infty)$, and

$$
\lim _{x \rightarrow 0} f(x)=1, \quad \lim _{x \rightarrow+\infty} f(x)=1
$$

Then, we have

$$
f(x) \leq 1
$$

If $p>1$, then we know $f(x)$ is monotone increasing on $(0,1]$ and monotone decreasing on $[1,+\infty)$. So, we have

$$
f(x) \leq f(1)=2^{p-1}
$$

The proof is completed.

Next, we have the following theorem concerning blow up.

Theorem 2.6. Assume (G1), (G2), (G3) and (2.1) hold. Let $m \geq 2, p>\max \{2, m\}$ satisfy (2.1). Assume further that

$$
\int_{0}^{+\infty} g(s) \mathrm{d} s<\frac{\frac{p}{2}-1}{\frac{p}{2}-1+\frac{1}{2 p}}=1-\frac{1}{(p-1)^{2}}
$$


if $\left(u_{0}, u_{1}\right) \in H_{0}^{1}(\Omega) \times L^{2}(\Omega)$ and satisfy $E(0)<0$, then the solution of problem(1.10)blow up in finite time.

Proof. From (2.2), we have

$$
H^{\prime}(t)=-E^{\prime}(t) \geq \frac{\xi}{2}\|\eta\|_{g, V}^{2}+\varepsilon_{1}\left\|\nabla u_{t}\right\|_{2}^{2}+\varepsilon_{2}\left\|u_{t}\right\|_{m}^{m} \geq 0
$$

consequently, we have

$$
0<H(0) \leq H(t) \leq \frac{\varepsilon_{3}}{p}\|u\|_{p}^{p}
$$

Similar to [18], then we define the weighed functional

$$
W(t)=H^{1-\alpha}(t)+\varepsilon \int_{\Omega} u u_{t} \mathrm{~d} x
$$

where $\varepsilon>0$ shall be chosen in what follows. Let

$$
0<\alpha<\min \left\{\frac{p-2}{2 p}, \frac{p-m}{p(m-1)}\right\}
$$

By multiplying (1.10) by $u$ and taking a derivative of (2.17), we obtain

$$
\begin{aligned}
W^{\prime}(t) \geq & (1-\alpha) H^{-\alpha}(t) H^{\prime}(t)+\varepsilon \int_{\Omega}\left|u_{t}\right|^{2} \mathrm{~d} x+\varepsilon \int_{\Omega} u u_{t t} \mathrm{~d} x \\
\geq & (1-\alpha) H^{-\alpha}(t) H^{\prime}(t)+\varepsilon\left\|u_{t}\right\|_{2}^{2}-\varepsilon\left(1-\int_{0}^{+\infty} g(s) \mathrm{d} s\right)\|\nabla u\|_{2}^{2}+\varepsilon \varepsilon_{3}\|u\|_{p}^{p} \\
& \quad-\varepsilon \int_{0}^{+\infty} g(s) \int_{\Omega} \nabla \eta(s) \nabla u(t) \mathrm{d} x \mathrm{~d} s-\varepsilon \varepsilon_{2} \int_{\Omega} u u_{t}\left|u_{t}\right|^{m-2} \mathrm{~d} x-\varepsilon \varepsilon_{1} \int_{\Omega} \nabla u_{t} \nabla u \mathrm{~d} x .
\end{aligned}
$$

By using Holder inequality and Young's inequality to estimate the fourth term on the right hand side of (2.19)

$$
\int_{0}^{+\infty} g(s) \int_{\Omega} \nabla \eta(s) \nabla u(t) \mathrm{d} x \mathrm{~d} s \leq \beta\|\eta\|_{g, V}^{2}+\frac{1}{4 \beta} \int_{0}^{+\infty} g(s) \mathrm{d} s\|\nabla u\|_{2}^{2}
$$

for some number $\beta$ with $0<\beta<\frac{p}{2}$. From (2.3) we have

$$
\varepsilon_{3}\|u\|_{p}^{p}=p H(t)+\frac{p}{2}\left\|u_{t}\right\|_{2}^{2}+\frac{p}{2}\left(1-\int_{0}^{+\infty} g(s) \mathrm{d} s\right)\|\nabla u\|_{2}^{2}+\frac{p}{2}\|\eta\|_{g, V}^{2}
$$

Then, we have

$$
\begin{aligned}
W^{\prime}(t) \geq & (1-\alpha) H^{-\alpha}(t)\left[\varepsilon_{2}\left\|u_{t}\right\|_{m}^{m}+\varepsilon_{1}\left\|\nabla u_{t}\right\|_{2}^{2}\right]+\varepsilon\left\|u_{t}\right\|_{2}^{2} \\
& -\varepsilon\left(1-\int_{0}^{+\infty} g(s) \mathrm{d} s\right)\|\nabla u\|_{2}^{2}-\varepsilon \beta\|\eta\|_{g, V}^{2}-\frac{\varepsilon}{4 \beta} \int_{0}^{+\infty} g(s) \mathrm{d} s\|\nabla u\|_{2}^{2}-\varepsilon \varepsilon_{1} \int_{\Omega} \nabla u_{t} \nabla u \mathrm{~d} x \\
& +\varepsilon\left[p H(t)+\frac{p}{2}\left\|u_{t}\right\|_{2}^{2}+\frac{p}{2}\left(1-\int_{0}^{+\infty} g(s) \mathrm{d} s\right)\|\nabla u\|_{2}^{2}+\frac{p}{2}\|\eta\|_{g, V}^{2}\right]-\varepsilon \varepsilon_{2} \int_{\Omega} u u_{t}\left|u_{t}\right|^{m-2} \mathrm{~d} x,
\end{aligned}
$$

that is

$$
\begin{aligned}
W^{\prime}(t) \geq & (1-\alpha) H^{-\alpha}(t)\left[\varepsilon_{2}\left\|u_{t}\right\|_{m}^{m}+\varepsilon_{1}\left\|\nabla u_{t}\right\|_{2}^{2}\right]+\varepsilon\left(\frac{p}{2}+1\right)\left\|u_{t}\right\|_{2}^{2} \\
& +\varepsilon\left(\frac{p}{2}-\beta\right)\|\eta\|_{g, V}^{2}+\varepsilon\left[\left(\frac{p}{2}-1\right)-\left(\frac{p}{2}-1+\frac{1}{4 \beta}\right) \int_{0}^{+\infty} g(s) \mathrm{d} s\right]\|\nabla u\|_{2}^{2} \\
& +\varepsilon p H(t)-\varepsilon \varepsilon_{1} \int_{\Omega} \nabla u_{t} \nabla u \mathrm{~d} x-\varepsilon \varepsilon_{2} \int_{\Omega} u u_{t}\left|u_{t}\right|^{m-2} \mathrm{~d} x .
\end{aligned}
$$

By using Holder inequality and Young's inequality to estimate the last two terms on right hand side of (2.24), we obtain 
and

$$
\begin{aligned}
\int_{\Omega} u u_{t}\left|u_{t}\right|^{m-2} \mathrm{~d} x & \leq\left(\int_{\Omega}|u|^{m} \mathrm{~d} x\right)^{\frac{1}{m}}\left(\int_{\Omega}\left(\left|u_{t}\right|^{m-1}\right)^{\frac{m}{m-1}} \mathrm{~d} x\right)^{\frac{m-1}{m}}=\|u\|_{m}\left\|u_{t}\right\|_{m}^{m-1} \\
& \leq \frac{\delta^{m}}{m}\|u\|_{m}^{m}+\frac{m-1}{m} \delta^{-\frac{m}{m-1}}\left\|u_{t}\right\|_{m}^{m},
\end{aligned}
$$

and

$$
\|u\|_{m}^{m} \leq\left(\int_{\Omega}\left(|u|^{m}\right)^{\frac{p}{m}} \mathrm{~d} x\right)^{\frac{m}{p}}\left(\int_{\Omega} 1 \mathrm{~d} x\right)^{1-\frac{m}{p}} \leq C\|u\|_{p}^{m}
$$

$$
\int_{\Omega} \nabla u_{t} \nabla u \mathrm{~d} x \leq \gamma\left\|\nabla u_{t}\right\|_{2}^{2}+\frac{1}{4 \gamma}\|\nabla u\|_{2}^{2}
$$

Substituting (2.24), (2.25) and (2.26) and to (2.23), we have

$$
\begin{aligned}
W^{\prime}(t) \geq & \varepsilon_{2}\left[(1-\alpha) H^{-\alpha}(t)-\frac{m-1}{m} \delta^{-\frac{m}{m-1}}\right]\left\|u_{t}\right\|_{m}^{m}+\varepsilon\left(\frac{p}{2}+1\right)\left\|u_{t}\right\|_{2}^{2} \\
& +\varepsilon_{1}\left[(1-\alpha) H^{-\alpha}(t)-\varepsilon \gamma\right]\left\|\nabla u_{t}\right\|_{2}^{2}+\varepsilon\left(\frac{p}{2}-\beta\right)\|\eta\|_{g, V}^{2} \\
& +\varepsilon\left[\left(\frac{p}{2}-1-\frac{\varepsilon_{1}}{4 \gamma}\right)-\left(\frac{p}{2}-1+\frac{1}{4 \beta}\right) \int_{0}^{+\infty} g(s) \mathrm{d} s\right]\|\nabla u\|_{2}^{2} \\
& +\varepsilon\left[p H(t)-C \varepsilon_{2} \frac{\delta^{m}}{m}\|u\|_{p}^{m}\right],
\end{aligned}
$$

by taking $\delta$ so that $\delta^{-\frac{m}{m-1}}=K H^{-\alpha}(t)$, so $\delta^{m}=K^{1-m} H^{\alpha(m-1)}(t)$, for large $K$ to be specified later, and substituting in (2.28) we obtain

$$
\begin{aligned}
W^{\prime}(t) \geq & \varepsilon_{2}\left[(1-\alpha)-\frac{m-1}{m} \varepsilon K\right] H^{-\alpha}(t)\left\|u_{t}\right\|_{m}^{m}+\varepsilon\left(\frac{p}{2}+1\right)\left\|u_{t}\right\|_{2}^{2} \\
& +\varepsilon_{1}\left[(1-\alpha) K^{-1} \delta^{-\frac{m}{m-1}}-\varepsilon \gamma\right]\left\|\nabla u_{t}\right\|_{2}^{2}+\varepsilon\left(\frac{p}{2}-\beta\right)\|\eta\|_{g, V}^{2} \\
& +\varepsilon\left[\left(\frac{p}{2}-1-\frac{\varepsilon_{1}}{4 \gamma}\right)-\left(\frac{p}{2}-1+\frac{1}{4 \beta}\right) \int_{0}^{+\infty} g(s) \mathrm{d} s\right]\|\nabla u\|_{2}^{2} \\
& +\varepsilon\left[p H(t)-C \varepsilon_{2} \frac{K^{1-m}}{m} H^{\alpha(m-1)}\|u\|_{p}^{m}\right],
\end{aligned}
$$

by taking proper $\varepsilon, \gamma, K$ such that

so, we have

$$
\begin{aligned}
& (1-\alpha)-\frac{m-1}{m} \varepsilon K \geq 0, \\
& (1-\alpha) K^{-1} \delta^{-\frac{m}{m-1}}-\varepsilon \gamma \geq 0,
\end{aligned}
$$

$$
\begin{aligned}
W^{\prime}(t) \geq & \varepsilon\left(\frac{p}{2}+1\right)\left\|u_{t}\right\|_{2}^{2}+\varepsilon\left(\frac{p}{2}-\beta\right)\|\eta\|_{g, V}^{2}+\varepsilon\left[\left(\frac{p}{2}-1-\frac{\varepsilon_{1}}{4 \gamma}\right)-\left(\frac{p}{2}-1+\frac{1}{4 \beta}\right) \int_{0}^{+\infty} g(s) \mathrm{d} s\right]\|\nabla u\|_{2}^{2} \\
& +\varepsilon\left[p H(t)-C \varepsilon_{2} \frac{K^{1-m}}{m} H^{\alpha(m-1)}\|u\|_{p}^{m}\right],
\end{aligned}
$$


From (2.16), we have

Then, hence (2.31) yields

$$
H^{\alpha(m-1)}\|u\|_{p}^{m} \leq\left(\frac{\varepsilon_{3}}{p}\right)^{\alpha(m-1)}\|u\|_{p}^{m+\alpha p(m-1)}
$$

$$
\begin{aligned}
W^{\prime}(t) \geq \varepsilon\left(\frac{p}{2}+1\right)\left\|u_{t}\right\|_{2}^{2}+\varepsilon a_{1}\|\eta\|_{g, V}^{2}+\varepsilon a_{2}\|\nabla u\|_{2}^{2} \\
+\varepsilon\left[p H(t)-C \varepsilon_{2} \frac{K^{1-m}}{m}\left(\frac{\varepsilon_{3}}{p}\right)^{\alpha(m-1)}\|u\|_{p}^{m+\alpha p(m-1)}\right]
\end{aligned}
$$

where $a_{1}=\frac{p}{2}-\beta, a_{2}=\left(\frac{p}{2}-1-\frac{\varepsilon_{1}}{4 \gamma}\right)-\left(\frac{p}{2}-1+\frac{1}{4 \beta}\right) \int_{0}^{+\infty} g(s) \mathrm{d} s$, and taking proper $\varepsilon_{1}, \beta, \gamma$ such that $a_{1}>0, a_{2}>0$.

Writing $s=m+\alpha p(m-1)$, for $0<\alpha<\min \left\{\frac{p-2}{2 p}, \frac{p-m}{p(m-1)}\right\}$, we know $0<s \leq p$. By using Corollary 2.4 we have

$$
\begin{aligned}
W^{\prime}(t) & \geq \varepsilon\left(\frac{p}{2}+1\right)\left\|u_{t}\right\|_{2}^{2}+\varepsilon a_{1}\|\eta\|_{g, V}^{2}+\varepsilon a_{2}\|\nabla u\|_{2}^{2} \\
& +\varepsilon\left[p H(t)-C_{1}\left(-H(t)-\left\|u_{t}\right\|_{2}^{2}-\|\eta\|_{g, V}^{2}+\|u\|_{p}^{p}\right)\right]
\end{aligned}
$$

where $C_{1}=C \varepsilon_{2} \frac{K^{1-m}}{m}\left(\frac{\varepsilon_{3}}{p}\right)^{\alpha(m-1)}$.

From (2.3) and (G1) we have

$$
H(t) \geq \frac{\varepsilon_{3}}{p}\|u\|_{p}^{p}-\frac{1}{2}\left\|u_{t}\right\|_{2}^{2}-\frac{1}{2}\|\nabla u\|_{2}^{2}-\frac{1}{2}\|\eta\|_{g, V}^{2}
$$

writing $p=2 a_{3}+\left(p-2 a_{3}\right)$, where $a_{3}=\min \left\{a_{1}, a_{2}\right\}$, estimate (2.34) yields

$$
\begin{aligned}
W^{\prime}(t) \geq & \varepsilon\left(\frac{p}{2}+1+C_{1}-a_{3}\right)\left\|u_{t}\right\|_{2}^{2}+\varepsilon\left(a_{1}+C_{1}-a_{3}\right)\|\eta\|_{g, V}^{2}+\varepsilon\left(a_{2}-a_{3}\right)\|\nabla u\|_{2}^{2} \\
& +\varepsilon\left(p-2 a_{3}+C_{1}\right) H(t)+\varepsilon\left(\frac{2 a_{3}}{p}-C_{1}\right)\|u\|_{p}^{p},
\end{aligned}
$$

at this point, we choose $K$ large enough, so $C_{1}$ is small enough. Then there exists $\sigma>0$ such that

$$
W^{\prime}(t) \geq \varepsilon \sigma\left(\left\|u_{t}\right\|_{2}^{2}+\|\eta\|_{g, V}^{2}+H(t)+\|u\|_{p}^{p}\right) \geq 0
$$

By using Holder inequality and Young's inequality, we next estimate

$$
\|u\|_{2}=\left(\int_{\Omega}|u|^{2} \mathrm{~d} x\right)^{\frac{1}{2}} \leq\left[\left(\int_{\Omega}\left(|u|^{2}\right)^{\frac{p}{2}} \mathrm{~d} x\right)^{\frac{2}{p}}\left(\int_{\Omega} 1 \mathrm{~d} x\right)^{1-\frac{2}{p}}\right]^{\frac{1}{2}} \leq C\|u\|_{p}
$$

and

which implies

$$
\left|\int_{\Omega} u u_{t} \mathrm{~d} x\right| \leq\|u\|_{2}\left\|u_{t}\right\|_{2} \leq C\|u\|_{p}\left\|u_{t}\right\|_{2}
$$

$$
\left|\int_{\Omega} u u_{t} \mathrm{~d} x\right|^{\frac{1}{1-\alpha}} \leq C\|u\|_{p}^{\frac{1}{1-\alpha}}\left\|u_{t}\right\|_{2}^{\frac{1}{1-\alpha}} \leq C\left(\|u\|_{p}^{\frac{\mu}{1-\alpha}}+\left\|u_{t}\right\|_{2}^{\frac{\theta}{1-\alpha}}\right)
$$


where $\frac{1}{\mu}+\frac{1}{\theta}=1$, we take $\theta=2(1-\alpha)$, to get $t=\frac{\mu}{1-\alpha}=\frac{2}{1-2 \alpha} \leq p$ by (2.18). We then use Corollary 2.4

$$
\begin{aligned}
\left|\int_{\Omega} u u_{t} \mathrm{~d} x\right|^{\frac{1}{1-\alpha}} & \leq C\left(\|u\|_{p}^{t}+\left\|u_{t}\right\|_{2}^{2}\right) \\
& \leq C\left(H(t)+\left\|u_{t}\right\|_{2}^{2}+\|u\|_{p}^{p}+\|\eta\|_{g, V}^{2}\right),
\end{aligned}
$$

By using $C_{p}$ inequality we have

$$
\begin{aligned}
W^{\frac{1}{1-\alpha}}(t) & =\left[H^{1-\alpha}(t)+\varepsilon \int_{\Omega} u u_{t} \mathrm{~d} x\right]^{\frac{1}{1-\alpha}} \\
& \leq 2^{\frac{1}{1-\alpha}}\left[H(t)+\left|\int_{\Omega} u u_{t} \mathrm{~d} x\right|^{\frac{1}{1-\alpha}}\right] \\
& \leq C\left[H(t)+\left\|u_{t}\right\|_{2}^{2}+\|u\|_{p}^{p}+\|\eta\|_{g, V}^{2}\right],
\end{aligned}
$$

According to (2.36) and (2.41), we get

$$
W^{\prime}(t) \geq k W^{\frac{1}{1-\alpha}}(t)
$$

where $k=C$. According to the theorem of Ordinary Differential Equation, we have

$$
W^{\frac{\alpha}{1-\alpha}}(t) \geq \frac{1}{W^{\frac{-\alpha}{1-\alpha}}(0)-\frac{k \alpha t}{1-\alpha}}
$$

So, we know $W(t)$ blow up in finite time $T^{*} \leq \frac{1-\alpha}{k \alpha W^{\frac{\alpha}{1-\alpha}}(0)}$. The proof is completed.

\section{Global Existence}

In this section, we show that solution of (1.10) is global if $m \geq p$.

Lemma 3.1. For $a \geq 0, x>0, f(x)=\frac{a^{x}}{x}$ is the convexity of the function.

Proof.

$$
f^{\prime \prime}(x)=\frac{a^{x}\left[(\ln a-1)^{2}+1\right]}{x^{3}}>0
$$

so, $f(x)$ is convex.

Theorem 3.2. Assume (G1), (G2) and (G3) hold. Let $p \leq m$ satisfy (2.1). If for any initial data $\left(u_{0}, u_{1}\right) \in H_{0}^{1}(\Omega) \times L^{2}(\Omega)$ with compact support, so problem (1.7) has a unique global solution, such that

$$
u \in C\left([0, T) ; H_{0}^{1}(\Omega)\right) \cap C^{1}\left([0, T) ; L^{2}(\Omega)\right)
$$

for any $T>0$.

Proof. Similar to [23], we set

$$
F(t)=\frac{1}{2}\left\|u_{t}\right\|_{2}^{2}+\frac{1}{2}\left(1-\int_{0}^{+\infty} g(s) \mathrm{d} s\right)\|\nabla u\|_{2}^{2}+\frac{1}{2}\|\eta\|_{g, V}^{2}+\frac{\varepsilon_{3}}{p}\|u\|_{p}^{p}
$$

from (2.3), we have

$$
F(t)=E(t)+\frac{2 \varepsilon_{3}}{p}\|u\|_{p}^{p}
$$


By differentiating $F(t)$ and using (2.2), we get

$$
\begin{aligned}
F^{\prime}(t) & =E^{\prime}(t)+2 \varepsilon_{3} \int_{\Omega}|u|^{p-2} u u_{t} \mathrm{~d} x \\
& \leq-\varepsilon_{1}\left\|\nabla u_{t}\right\|_{2}^{2}-\frac{\xi}{2}\|\eta\|_{g, V}^{2}-\varepsilon_{2}\left\|u_{t}\right\|_{m}^{m}+2 \varepsilon_{3} \int_{\Omega}|u|^{p-2} u u_{t} \mathrm{~d} x .
\end{aligned}
$$

By using Holder inequality and Young's inequality, we next estimate

$$
\begin{aligned}
\int_{\Omega}|u|^{p-2} u u_{t} \mathrm{~d} x & \leq\left(\int_{\Omega}\left|u_{t}\right|^{p} \mathrm{~d} x\right)^{\frac{1}{p}}\left(\int_{\Omega}\left(|u|^{p-1}\right)^{\frac{p}{p-1}} \mathrm{~d} x\right)^{\frac{p-1}{p}}=\left\|u_{t}\right\|_{p}\|u\|_{p}^{p-1} \\
& \leq \frac{1}{p}\left\|u_{t}\right\|_{p}^{p}+\frac{p-1}{p}\|u\|_{p}^{p} \leq \frac{1}{p}\left\|u_{t}\right\|_{p}^{p}+\|u\|_{p}^{p} .
\end{aligned}
$$

Setting $(y)=\frac{\int_{\Omega}\left|u_{t}\right|^{y} \mathrm{~d} x}{y}=\frac{\left\|u_{t}\right\|_{y}^{y}}{y}$, we know $f(y)$ is the convexity of function by Corollary 3.1. Since $2<p$ $\leq m$, we obtain

$$
\frac{\left\|u_{t}\right\|_{p}^{p}}{p} \leq \frac{\left\|u_{t}\right\|_{2}^{2}}{2}+\frac{\left\|u_{t}\right\|_{m}^{m}}{m}
$$

Substituting (3.5) to (3.3), we have

$$
F^{\prime}(t) \leq-\varepsilon_{1}\left\|\nabla u_{t}\right\|_{2}^{2}-\frac{\xi}{2}\|\eta\|_{g, V}^{2}-\varepsilon_{2}\left\|u_{t}\right\|_{m}^{m}+2 \varepsilon_{3}\left(\frac{\left\|u_{t}\right\|_{2}^{2}}{2}+\frac{\left\|u_{t}\right\|_{m}^{m}}{m}+\|u\|_{p}^{p}\right)
$$

so, there exists a small enough constant $C$ such that

$$
F^{\prime}(t) \leq C F(t)
$$

Then, by using Gronwall inequality and continuation principle, we complete the proof of the global existence result.

\section{Acknowledgements}

The authors express their sincere thanks to the anonymous reviewer for his/her careful reading of the paper, giving valuable comments and suggestions. These contributions greatly improved the paper.

This work is supported by the National Natural Sciences Foundation of People's Republic of China under Grant 11161057

\section{References}

[1] Messaoudi, S.A. (2003) Blow Up and Global Existence in a Nonlinear Viscoelastic Wave Equation. Mathematische Nachrichten, 260, 58-66. http://dx.doi.org/10.1002/mana.200310104

[2] Messaoudi, S.A. (2006) Blow Up of Positive-Initial-Energy Solutions of a Nonlinear Viscoelastic Hyperbolic Equation. Journal of Mathematical Analysis and Applications, 320, 902-915. http://dx.doi.org/10.1016/j.jmaa.2005.07.022

[3] Wu, S.T. (2006) Blow-Up of Solutions for an Integro-Differential Equation with a Nonlinear Source. Electronic Journal of Differential Equations, 45, 1-9.

[4] Kafini, M., Messaoudi, S.A. (2008) A Blow-Up Result in a Cauchy Viscoelastic Problem. Applied Mathematics Letters, 21, 549-553. http://dx.doi.org/10.1016/j.aml.2007.07.004

[5] Ball, J. (1977) Remarks on Blow-Up and Nonexistence Theorems for Nonlinear Evolutions Equation. Quarterly Journal of Mathematics, 28, 473-486. http://dx.doi.org/10.1093/qmath/28.4.473

[6] Haraux, A. and Zuazua, E. (1988) Decay Estimates for Some Semilinear Damped Hyperbolic Problems. Archive for Rational Mechanics and Analysis, 100, 191-206. http://dx.doi.org/10.1007/BF00282203

[7] Levine, H.A. (1974) Instability and Nonexistence of Global Solutions of Nonlinear Wave Equation of the Form $P u_{t t}=$ $A u+F(u)$. Transactions of the American Mathematical Society, 192, 1-21. 
[8] Levine, H.A. (1974) Some Additional Remarks on the Nonexistence of Global Solutions to Nonlinear Wave Equation. SIAM Journal on Mathematical Analysis, 5, 138-146. http://dx.doi.org/10.1137/0505015

[9] Georgiev, V. and Todorova, G. (1994) Existence of Solutions of the Wave Equation with Nonlinear Damping and Source Terms. Journal of Differential Equations, 109, 295-308. http://dx.doi.org/10.1006/jdeq.1994.1051

[10] Messaoudi, S.A. (2001) Blow Up in a Nonlinearly Damped Wave Equation. Mathematische Nachrichten, 231, $105-$ 111. http://dx.doi.org/10.1002/1522-2616(200111)231:1<105::AID-MANA105>3.0.CO;2-I

[11] Berrimi, S. and Messaoudi, S.A. (2006) Existence and Decay of Solutions of a Viscoelastic Equation with a Nonlinear Source. Nonlinear Analysis, 64, 2314-2331. http://dx.doi.org/10.1016/j.na.2005.08.015

[12] Song, H.T. and Xue, D.S. (2014) Blow up in a Nonlinear Viscoelastic Wave Equation with Strong Damping. Nonlinear Analysis, 109, 245-251. http://dx.doi.org/10.1016/j.na.2014.06.012

[13] Song, H.T. and Zhong, C.K. (2010) Blow-Up of Solutions of a Nonlinear Viscoelastic Wave Equation. Nonlinear Analysis: Real World Applications, 11, 3877-3883. http://dx.doi.org/10.1016/j.nonrwa.2010.02.015

[14] Lu, L.Q. and Li, S.J. (2011) Cauchy Problem for a Nonlinear Viscoelastic Equation with Nonlinear Damping and Source Term. Applied Mathematics Letters, 24, 1275-1281. http://dx.doi.org/10.1016/j.aml.2011.01.009

[15] Kafini, M. and Mustafa, M.I. (2014) Blow-Up Result in a Cauchy Viscoelastic Problem with Strong Damping and Dispersive. Nonlinear Analysis: Real World Applications, 20, 14-20. http://dx.doi.org/10.1016/j.nonrwa.2014.04.005

[16] Kafini, M. and Messaoudi, S.A. (2008) A Blow-Up Result in a Cauchy Viscoelastic Problem. Applied Mathematics Letters, 21, 549-553. http://dx.doi.org/10.1016/j.aml.2007.07.004

[17] Cavalcanti, M.M., Domingos Cavalcanti, V.N. and Soriano, J.A. (2002) Exponential Decay for the Solution of Semilinear Viscoelastic Wave Equation with Localized Damping. Electronic Journal of Differential Equations, 2002, 1-14.

[18] Cavalcanti, M.M., Domingos Cavalcanti, V.N. and Ferreira, J. (2001) Existence and Uniform Decay for Nonlinear Viscoelastic Equation with Strong Damping. Mathematical Methods in the Applied Sciences, 24, 1043-1053. http://dx.doi.org/10.1002/mma.250

[19] Qin, Y.M., Feng, B.W. and Zhang, M. (2014) Uniform Attractors for a Non-Autonomous Viscoelastic Equation with a Past History. Nonlinear Analysis, 101, 1-15. http://dx.doi.org/10.1016/j.na.2014.01.006

[20] Conti, M., Marchini, E.M. and Pata, V. (2014) A Well Posedness Result for Nonlinear Viscoelastic Equations with Memory. Nonlinear Analysis, 94, 206-216. http://dx.doi.org/10.1016/j.na.2013.08.015

[21] Zhou, Y. (2005) A Blow-Up Result for a Nonlinear Wave Equation with Damping and Vanishing Initial Energy in $R^{N}$. Applied Mathematics Letters, 18, 281-286. http://dx.doi.org/10.1016/j.aml.2003.07.018

[22] Cavalcanti, M.M., Domingos Cavalcanti, V.N. and Soriano, J.A. (2002) Global Existence and Asymptotic Stability for Viscoelastic Problems. Differential Integral Equations, 15, 731-748.

[23] Lu, L.Q. and Li, S.J. (2011) Cauchy Problem for a Nonlinear Viscoelastic Equation with Nonlinear Damping and Source Term. Applied Mathematics Letters, 24, 1275-1281. http://dx.doi.org/10.1016/j.aml.2011.01.009 